\title{
Avaliação da qualidade do ar interior: um estudo de caso em um frigorífico no sul do Estado do Espírito Santo
}

\author{
Wanderson Lyrio Bermudes \\ Doutor em Ciências Florestais (UFES) \\ $\triangle$ wanderson.bermudes@hotmail.com \\ Denis Ferreira Coutinho \\ Higienista Ocupacional \\ $\bowtie$ ana.pereira@uerj.br
}

Recebido em 12 de janeiro de 2020

Aceito em 24 de abril de 2020

\begin{abstract}
Resumo:
A necessidade de garantir uma qualidade de ar em ambiente interno climatizado, vem ampliando as ações de fiscalização e melhoria. Esse estudo de caso apresenta a avaliação da qualidade de ar emum frigorífico localizado no sul do Estado do Espírito Santo, em 2015, utilizando a metodologia descrita na Resolução 09 de 2003 da Agência Nacional de Vigilância Sanitária - ANVISA, com análise dos agentes: partículas fúngicas no ar, dióxido de carbono, temperatura, umidade e velocidade do ar. Dentre os resultados a presentados, foi observado no setor de desossa valor de dióxido de carbono de 1.720 ppm, superior ao recomendado, que apesar de não apresentar grandes problemas de toxicidade, causa no trabalhador a sensação de não haver ar suficiente. 0 resultado de temperatura e umidade apresentaram valores fora da faixa recomendável, devido à particularidade da empresa e atividade que necessita de menor temperatura para conservação dos alimentos. $O$ resultado do parâmetro da contagem de fungos e relação do ar interno e externo para a concentração de partículas fungicidasno ambiente foi indicado como satisfatório. Diante desse resultado sugere de forma preventiva uma maior troca de ar do ambiente de forma a tornar mais satisfatória a presença do trabalhador no ambiente, devido a presença de dióxido de carbono que pode sofrer alteração ao longo do dia.
\end{abstract}

Palavras-chave: Ambiente laboral, Qualidade do ar, Saúde do trabalhador, Vigilância Sanitária.

\section{Indoor air quality assessment: a case study in a refrigerator in the southern state of Espírito Santo}

\begin{abstract}
:
The need to guarantee a quality of the indoor climate, has been increasing as inspection and improvement actions. This case study presents an assessment of air quality in the refrigerator located in the State of Espírito Santo, in 2015, using an application methodology in Resolution 09 of 2003 of the National Health Surveillance Agency - ANVISA, with analysis of the agents: fungal in the air , carbon dioxide, temperature, humidity and air speed. Among the results presented, a carbon dioxide value of $1,720 \mathrm{ppm}$ was observed in the sector, higher than recommended, which, despite not presenting major toxicity problems, causes the worker to feel that there is not enough. The temperature result and the values indicated for the recommended range, due to the particularity of the company and activity, are the ones that require lower temperature for food preservation. The result of the fungus count parameter and the internal and external relationship for the concentr ation of fungicides in the environment was indicated as satisfactory. In view of this result, it is suggested that a greater preventive way of exchanging the environment in order to make the presence of the
\end{abstract}


worker in the environment more satisfactory, due to the presence of carbon dioxide that can suffer damage throughout the day.

Keywords: Work environment, Air quality, Worker's health, Health Surveillance.

\section{Evaluación de la calidad del aire interior: un estudio de caso en un frigorífico en el sur del Estado de Espírito Santo}

\section{Resumen:}

La necesidad de garantizar la calidad del aire en un ambiente interior con aire acondicionado ha aumentado las acciones de inspección y mejora. Este estudio de caso presenta la evaluación de la calidad del aire en un refrigerador ubicado en el sur del Estado de Espírito Santo, en 2015, utilizando la metodología descrita en la Resolución 09 de 2003 de la Agencia Nacional de Vigilancia Sanitaria ANVISA, con análisis de los agentes: partículas hongos en el aire, dióxido de carbono, temperatura, humedad y velocidad del aire. Entre los resultados presentados, se observó en el sector de deshuesado un valor de dióxido de carbono de 1.720 ppm, superior al recomendado, que, a pesar de no presentar problemas de toxicidad importantes, hace que el trabajador sienta que no hay suficiente aire. Los resultados de temperatura y humedad mostraron valores fuera del rango recomendado, debido a la particularidad de la empresa y la actividad que requiere una temperatura más baja para la conservación de los alimentos. El resultado del parámetro de recuento de hongos y la relación del aire interior y exterior a la concentración de partículas fungicidas en el medio ambiente se indicó como satisfactoria. En vista de este resultado, sugiere de manera preventiva un mayor intercambio de aire en el ambiente para hacer que la presencia del trabajador en el ambiente sea más satisfactoria, debido a la presencia de dióxido de carbono que puede cambiar durante el día.

Palabras clave: Ambiente de trabajo, Calidad del aire Salud del trabajador, Vigilancia sanitaria.

\section{INTRODUÇÃO}

A saúde e o bem-estar dos ocupantes de edifícios, especialmente aqueles designados para uso comercial e administrativo, vem ao longo dos anos, devido a doenças geradas nesses ambientes e a evolução do conhecimento sobre o ambiente interno climatizados, ampliando as intervenções de melhorias e fiscalização (COSTA Maria; COSTA Marco, 2006).

No contexto histórico, em 1976 no estado da Filadélfia nos Estados Unidos, na estação do verão, ocorreu um grande surto de contaminação respiratória, período no qual foram registrados 182 casos de hospitalizações com pneumonia grave e 29 óbitos entre os participantes da convenção dos Legionários, causado por infecção de bactéria, que posteriormente foi denominada de Legionella pneumophila. Nesse ocorrido foi confirmada a relação entre a doença e as condições do ambiente em que havia pouca renovação de ar interno, reconhecendo em 1982 a Síndrome do Edifício Doente comprovada pela contaminação do ar interno em ambiente climatizado (LEMOS, 2011).

No Brasil, este tema ganhou destaque em 1998, com o falecimento do então Ministro das Comunicações, Sérgio Motta, devido a complicações relativas a uma fibrose associada a 
infecções e inflamações no pulmão. Entre as causas apontadas estava a pré-disposição hereditária, a exposição a ambientes contaminados por bactérias instaladas em arcondicionado (CASTRO, 2007; INMETRO, 2002).

Tal fato e o contexto histórico, estimularam no Brasil o surgimento, em 28 de agosto de 1998, da Portaria número 3.523, da Agência Nacional de Vigilância Sanitária - ANVISA, que aprovou o regulamento técnico indicando medidas básicas referentes aos procedimentos de verificação visual do estado de limpeza, remoção de sujidades por métodos físicos e manutenção de todos os componentes dos sistemas de climatização, de modo a garantir a qualidade do ar de interiores e prevenção de riscos à saúde dos ocupantes de ambientes climatizados (BRASIL, 1998).

Em 24 de Outubro de 2000 foi constituída a Resolução número 176, sobre padrões referenciais de qualidade do ar interior em ambientes climatizados artificialmente de uso público e coletivo, e após o conhecimento e a experiência adquiridos no país nos dois anos de sua vigência, a mesma foi atualizada por meio da Resolução 09 de 16 de janeiro de 2003 (BRASIL, 2000; 2003).

No estado do Espírito Santo, dentre as diversas atividades econômicas existentes, destaca-se, nessa pesquisa o setor de abate de reses, que consiste em animais quadrúpedes, abatidos para alimentação humana. No Brasil o segmento merece grande destaque e alcançou em 2016 o abate de 29.668.976 bovinos, devido às vantagens como grandes dimensões de terra e condições climáticas (AURÉLIO NETO, 2018).

A atividade de abate de reses comporta a exposição a fatores de risco ocupacional que podem determinar efeitos adversos para a saúde de quem desenvolve a sua atividade profissional (ARAÚJO et al., 2013). Destaca-se nesse ambiente os riscos de postura inadequada, esforço repetitivo, risco biológico, acidentes com máquinas perfuro cortante, poucas pausas, que podem causar doenças por esforços repetitivos, lesões diversas, doenças das vias respiratórias superiores, reumáticas (ARAÚJO; GOSLING, 2008; SANTANA; RODRIGUES, 2014).

Dentre os diversos riscos presente no ambiente essa pesquisa tem como objetivo demonstrar a qualidade do ar no ambiente de trabalho interno em uma atividade de abate de reses no sul do Estado do Espírito Santo, conforme a Resolução 09 de 2003 da Anvisa (BRASIL, 2003). Uma vez que a exposição a esses riscos causa diversos males ao trabalhador, como doenças infecciosas e alérgicas, provocadas por toxinas produzidas pelos microorganismos 
que crescem nos sistemas de ventilação ocasionando a elevação da taxa de absenteísmo e a redução produtividade do trabalhador (CAMPOS et al., 2017; CASTRO, 2007; SCHIRMER, et al., 2011).

\section{METODOLOGIA}

O frigorífico em estudo está situado no sul do estado de Espírito Santo, com subclasse CNAE 10.11.2-5 Matadouro - abate de reses sob contrato - exceto abate de suínos, separados em cincos ambientes: desossa, expedição, anexo de carregamento e ponto externo (ATLAS, 2019). Os ambientes avaliados eram climatizados artificialmente, exceto o ponto externo e possuíam área inferior a mil metros quadrados, sendo, portanto, a necessidade de apenas uma avaliação por ambiente (BRASIL, 2003).

As avaliações foram realizadas, por empresa especializada disponibilizando os dados para esse estudo, na estação do verão no sul do Estado do Espírito Santo, em 15 de março de 2015, e complementaram, conforme Resolução 09 (BRASIL, 2003) os seguintes agentes: partículas fúngicas no ar, dióxido de carbono $-\mathrm{CO}_{2}$, temperatura, umidade e velocidade do ar. As avaliações de aerodispersóides não foram contempladas nessa pesquisa por se tratar de um ambiente frigorificado com elevada umidade, impossibilitando a coleta. Foi realizada uma avaliação por local de trabalho, pois cada ambiente avaliado tem área inferior até $1.000 \mathrm{~m}^{2}$.

Os agentes foram coletados com amostradores, conforme métodos de amostragem e marcadores (Quadro 1) descritos na Resolução 09 da Avisa, utilizando equipamentos aferidos antes e depois da avaliação, posicionados sobre um tripé metálico a uma altura de aproximadamente de 1,50 metros do piso, considerada a zona respiratória dos trabalhadores e em horários de pico de utilização do local (BRASIL, 2003). 
Quadro 1 - Método de amostragem e marcadores de agentes ambientais conforme Resolução 09 da Anvisa

\begin{tabular}{|c|c|}
\hline AGENTE & $\begin{array}{l}\text { MÉTODO DE AMOSTRAGEM, MARCADORES E } \\
\text { EQUIPAMENTOS UTILIZADOS }\end{array}$ \\
\hline $\begin{array}{l}\text { Partículas fúngicas } \\
\text { no ar }\end{array}$ & $\begin{array}{l}\text { Amostrador de ar por impactação com acelerador linear. } \\
\text { Marcador: Fungos viáveis expresso por Unidade de Formação } \\
\text { de Colônia por metro cúbico - ufc. } \mathrm{m}^{-3} \text {. Bomba EMS } 6025 \mathrm{SE}-\mathrm{V} \text {, } \\
\text { com impactador de bioaerosol. }\end{array}$ \\
\hline $\begin{array}{l}\text { Dióxido de carbono } \\
\left(\mathrm{CO}_{2}\right)\end{array}$ & $\begin{array}{l}\text { Equipamento de leitura direta. Marcador: Dióxido de carbono } \\
-\mathrm{CO}_{2} \text { expresso em Partes Por Milhão - ppm. Medidor de } \\
\text { Dióxido de Carbono, TSI } 7515 \text { e Bomba de Amostragem Marca } \\
\text { Air One, modelo TL-004. }\end{array}$ \\
\hline Temperatura & $\begin{array}{l}\text { Equipamentos de leitura direta. Termo-higrômetro. } \\
\text { Marcador: Temperatura do ar expresso em grau Celsius }-{ }^{\circ} \mathrm{C} \\
\text { avaliado com termômetros de bulbo seco. } \\
\text { Termoanemômetro de fio quente ITAN 800, marca } \\
\text { Instrutemp e Termohigrômetro HT } 270 \text {, marca Instrutherm. }\end{array}$ \\
\hline Umidade & $\begin{array}{l}\text { Equipamentos de leitura direta. Termo-higrômetro. } \\
\text { Marcador: a relação entre a quantidade de água existente } \\
\text { no ar e a quantidade máxima que poderia haver na mesma } \\
\text { temperatura expressa em percentagem - \%. } \\
\text { Termoanemômetro de fio quente ITAN 800, marca } \\
\text { Instrutemp e Termohigrômetro HT } 270 \text {, marca Instrutherm. }\end{array}$ \\
\hline Velocidade do ar & $\begin{array}{l}\text { Equipamentos de leitura direta. Anemômetro. Marcador: } \\
\text { Velocidade do ar expresso em metros por segundo }-\mathrm{m} \cdot \mathrm{s}^{-1} \text {. } \\
\text { Termoanemômetro de fio quente ITAN } 800 \text {, marca } \\
\text { Instrutemp e } \\
\text { Termohigrômetro HT } 270 \text {, marca Instrutherm. }\end{array}$ \\
\hline
\end{tabular}

Fonte: BRASIL, 2003.

Os resultados das avaliações foram comparados com o Valor Máximo de Recomendável - VMR, que são definidos como o valor limite recomendável que separa as condições de ausência e de presença do risco de agressão à saúde humana, conforme apresentado no Quadro 2. 
Quadro 2 - Padrões estabelecidos como Valor Máximo Recomendável - VMR conforme Resolução 09

\begin{tabular}{|c|c|c|}
\hline AGENTE & VMR & UNIDADE \\
\hline Partículas fúngicas no ar & Menor de 750 & ufc.m $\mathrm{m}^{-3}$ \\
\hline $\begin{array}{l}\text { Partículas fúngicas no ar para a } \\
\text { relação Interno - I / Externo - E. }\end{array}$ & Menor de 1,5 & - \\
\hline Dióxido de carbono $\mathrm{CO}_{2}$ & Menor de 1000 & ppm \\
\hline Temperatura no verão & Faixa recomendável - 23 a $26^{*}$ & ${ }^{\circ} \mathrm{C}$ \\
\hline Umidade relativa & Faixa recomendável - 40 a $65^{*}$ & $(\%)$ \\
\hline velocidade do ar & Menor de 0,25 & $\mathrm{~m} \cdot \mathrm{s}^{-1}$ \\
\hline
\end{tabular}

* Conforme recomenda a Resolução 09 de 2003 da Anvisa (BRASIL, 2003) a seleção da faixa de temperatura e umidade depende da finalidade e do local da instalação, que nesse estudo trata-se de um frigorifico que possui temperatura e práticas de higiene próprias para conservação dos alimentos. A Resolução 09 de 2003 da ANVISA não possui regulamentação para atividade de abate de reses. Fonte: BRASIL, 2003.

\section{RESULTADOS E DISCUSSÃO}

Os resultados analíticos dos agentes avaliados estão separados por setor e classificados com parecer satisfatório, quando o resultado não ultrapassar ou estiver dentro da faixa recomendável do VMR e insatisfatório nos resultados acima ou fora da faixa recomendável do VMR, similar ao adotado por Cerqueira e Guimarães Filho (2017).

No que se refere ao parâmetro de contagem de fungos, no ambiente e a relação do ambiente interno e externo, agente esse capaz de causar diversos tipos de doenças, foram obtidos os resultados conforme Tabela 1.

Tabela 1 - Resultado do parâmetro da contagem de fungos e relação do ar interno e externo para a concentração de partículas fungicidas no ambiente com parecer conforme VMR

\begin{tabular}{l|l|l|l}
\hline LOCAL & $\begin{array}{l}\text { CONCENTRAÇÃO DE } \\
\text { PARTÍCULAS FÚNGICAS } \\
\text { NO AMBIENTE }\left(U^{-3} C \text { M }^{-3}\right)\end{array}$ & $\begin{array}{l}\text { RELAÇÃO DO AR } \\
\text { INTERNO COMO } \\
\text { PONTO EXTERNO } \\
(\mathrm{I} / \mathrm{E})\end{array}$ & PARECER \\
\hline Desossa & 450 & $450 / 655=0,68$ & Satisfatório \\
\hline Expedição & 540 & $540 / 655=0,82$ & Satisfatório \\
\hline $\begin{array}{l}\text { Anexo de } \\
\text { carregamento }\end{array}$ & 610 & $610 / 655=0,93$ & Satisfatório \\
\hline Ponto externo & 655 & --- & Satisfatório \\
\hline
\end{tabular}

Fonte: AUTOR, 2015 
Os resultados para concentração de partículas fúngicas no ambiente e a relação do ar interno e externo para o mesmo agente foi inferior ao VMR (Tabela 1), portanto satisfatório conforme Resolução 09 da ANVISA, apesar do local com elevada umidade favorecer a proliferação desse agente que causam alergias e outros problemas respiratórios nos ocupantes (BRASIL, 2003; FERREIRA et al., 2012).

Os resultados inferiores ao VMR reduzem a possibilidade de doenças respiratórias, alérgicas ou infecciosas, devendo ser mantida a limpeza ou desinfecção dos filtros de ar condicionados e dos aparelhos de refrigeração (CARTAXO et al., 2007; CERQUEIRA; GUIMARÃES FILHO, 2017). Mesmo com o resultado inferior ao VMR, a Resolução 09 Anvisa (BRASIL, 2003) descreve como inaceitável a presença de fungos patogênicos e toxicológicos no ambiente, não avaliada nessa pesquisa.

Outro agente poluente do ar interno, porém de origem não biológica é o dióxido de carbono, que apesar de não apresentar grandes problemas de toxicidade, causa no trabalhador a sensação de não haver ar suficiente, além de conduzir a desmineralização dos ossos em exposições continuas (SCHIRMER et al., 2011).

A avaliação do dióxido de carbono - $\mathrm{CO}_{2}$, apresentada na Tabela 2, foi realizada em quatro setores da empresa em estudo: desossa, expedição e anexo ao carregamento. 0 ponto externo foi avaliado apenas para comparação da concentração de partículas fúngicas no ambiente interno e externo (CASTRO, 2007).

Tabela 2 - Resultado do parâmetro da contagem de fungos e a relação ambiente interno e externo

\begin{tabular}{l|l|l}
\hline LOCAL & CONCENTRAÇÃO DE $\mathrm{CO}_{2}$ PPM & PARECER \\
\hline Desossa & 1720 & Insatisfatório \\
\hline Expedição & 310 & Satisfatório \\
\hline Anexo de carregamento & 750 & Satisfatório \\
\hline
\end{tabular}

Fonte: AUTOR, 2015.

Os resultados apresentados na Tabela 2 indicam que no setor de desossa há uma elevada exposição a dióxido de carbono, com 720 ppm acima do VMR, que nessa atividade pode ser oriundo de processos metabólicos, desenvolvidos pelos ocupantes. Quando comparada a outros ambientes, como por exemplo uma escola, percebe-se que esse valor se 
aproxima quando ocorre grande aglomeração nesses ambientes escolares, conforme estudo de em Fakhoury (2017) escola de engenharia de São Paulo.

A exposição acima do VMR deve-se à falha inadequada das trocas de ar (MENEGALI, 2009). Portanto nesse contexto é necessário ampliar as medidas de troca de ar com exaustores apropriados para a atividade de abate de reses.

As avaliações de análises físicas dos agentes temperatura, umidade relativa e velocidade do ar com resultados apresentados na Tabela 3, tem como objetivo verificar o conforto térmico proporcionados aos trabalhadores.

Tabela 3 - Resultados dos parâmetros físicos: temperatura, umidade relativa e velocidade do ar

\begin{tabular}{l|l|l|l|l}
\hline \multirow{2}{*}{ LOCAL } & \multicolumn{2}{|l|}{ PARÁMETROS FÍSICO } & \multirow{2}{*}{ PARECER } \\
\cline { 2 - 4 } & Temperatura & Umidade & Velocidade do ar & \\
\hline Desossa & $19,9^{\circ} \mathrm{C}$ & $68 \%$ & $0,0 \mathrm{~m} \cdot \mathrm{s}^{-1}$ & Insatisfatório* \\
\hline Expedição & $17,2^{\circ} \mathrm{C}$ & $86 \%$ & $0,01 \mathrm{~m} \cdot \mathrm{s}^{-1}$ & Insatisfatório* \\
\hline $\begin{array}{l}\text { Anexo de } \\
\text { carregamento }\end{array}$ & $18,4^{\circ} \mathrm{C}$ & $80 \%$ & $0,0 \mathrm{~m} \cdot \mathrm{s}^{-1}$ & Insatisfatório* \\
\hline
\end{tabular}

* Conforme recomenda a Resolução 09 de 2003 da Anvisa (BRASIL, 2003) A seleção da faixa de temperatura e umidade depende da finalidade e do local da instalação, que nesse estudo trata-se de um frigorifico que possui temperatura e práticas de higiene próprias para conservação dos alimentos. A Resolução 09 de 2003 da ANVISA não possui regulamentação para atividade de abate de reses. Fonte: AUTOR, 2015

A análise de temperatura e umidade, conforme apresentado na Tabela 3, não pode ser o único critério para verificação dos índices de VMR, pois tratando-se de um frigorifico a temperatura e práticas de higiene são próprias para a conservação de alimentos (BAASCH; HASSE; ALEXANDRINI, 2009). No que se refere a velocidade do ar os resultados ficaram inferior ao VMR, indicando pouca renovação de ar.

\section{CONCLUSÃO}

Os resultados obtidos nessa pesquisa permitem concluir que a emissão de dióxido de carbono no setor de desossa está acima do permitido, sendo imprescindível a adoção de medidas para melhorar a qualidade do ambiente através de métodos de troca do ar. 
No que se refere a temperatura e umidade, apesar dos valores avaliados estarem fora do Valor Máximo de Recomendável, deve-se considerar a característica da atividade que exige cuidados especiais na conservação do alimento no abate de reses.

Deve-se ainda promover estudos multidisciplinares por parte da empresa e órgãos normativos e fiscalizadores, com o intuito de adequar o ambiente de trabalho as necessidades do trabalhador e estabelecer normativas aplicadas a ambientes especiais, como no caso de frigoríficos, para melhor fiscalização, tendo em vista que a avaliação da qualidade do ar interno não está contemplado em normativas do governo que tratam exclusivamente da proteção do trabalhador.

\section{REFERÊNCIAS}

ARAÚJO, G. C. D.; GOSLING, M. Gestão de acidentes de trabalho em uma empresa frigorífica: um estudo de caso. Revista Pretexto, v. 9, n. 1, 2008.

ARAÚJO, A. F. D. V.; ZANNONI, C.; LIMA, D. B. S.; SANTOS, E. A. dos.; DIAS, I. C. L. D.; RODRIGUES, Z. M. R.. Identificação de fatores de riscos ocupacionais no processo de abate de bovinos. Cadernos de Pesquisa, v. 19, n. 3, 2013.

ATLAS. Segurança e medicina do trabalho. Editora Altas. 82 Edição. São Paulo. 2019. 1.224 p.

AURÉLIO NETO, O. P. Estratégia espacial no mercado mundial de carne: a internacionalização do setor frigorífico brasileiro. Tese (Doutorado) - Universidade Federal de Goiás, Instituto de Estudos Socioambientais (Iesa), Programa de Pós-Graduação em Geografia, Goiânia, - 2018. 380 f.: il.

BAASCH, C. A.; HASSE, C. R.; ALEXANDRINI, F. Monitoração do controle de temperaturas de frigorífico conforme circular 175 do MAPA. SEGeT - Simpósio de Excelência em Gestão e Tecnologia. 2009

BRASIL. Agência Nacional de Vigilância Sanitária - ANVISA. Portaria no 3.523, de 28 de agosto de 1998. Aprova regulamento técnico que garante a qualidade do ar de interiores e prevenção de riscos à saúde dos ocupantes de ambientes climatizados. Brasília (DF): Diário Oficial da União; 31 ago. 1998.

BRASIL. Ministério da Saúde n. -176 de 24 de outubro de 2000. Determi na a publicação de orientação técnica elaborada por grupo técnico assessor, sobre padrões referenciais de qualidade do ar interior, em ambiente climatizados artificialmente de uso público e coletivo. Brasília, DF, 2000.

BRASIL. Agência Nacional de Vigilância Sanitária - ANVISA. Resolução 09, de 16 de janeiro de 2003. Determinar a publicação de orientação técnica elaborar por grupo técnico assessor, sobre padrões referenciais de qualidade do ar interior, em ambientes climatizados artificialmente de uso público e coletivo em anexo. Diário oficial da União, Brasília (DF), 20 jan. 2003.

CAMPOS, F. de M.; GOLIN, R.; CAIXETA, F. C.; SANCHES, L.; CAIXETA, D. S. Avaliação Quanti-Qualitativa do Ar Interior de Uma Biblioteca Pública do Município de Cuiabá-MT. E\&S Engineering and Science, v. 6, n. 1, p. 95-105, 2017. 
CARTAXO, E. F.; GONÇALVES, A. C. L. C.; COSTA, F. R.; COElHO, I. M. V.; SANTOS, J. G. dos. Aspectos de contaminação biológica em filtros de condicionadores de ar instalados em domicílios da cidade de Manaus - AM. Revista Engenharia Sanitária e Ambi ental. Rio de Janeiro, v. 12, n. 2, p. 202-211, 2007.

CASTRO, K. D. de. Impactos da qualidade do espaço arquitetônico na produtividade do trabalhador. Revista Pretexto, v. 8, n. 2, p. $23-38$, jul-dez. 2007.

CERQUEIRA, P. E. S; GUIMARÃES FILHO, A. B. Qualidade do ar em ambientes internos climatizados em uma indústria petroquímica. Revista Cientefico, v. 17, n. 35, p. 109-126, 2017.

COSTA, Maria. de F. B. da; COSTA, Marco. A. F. A qualidade do ar de interiores e a saúde humana. InterfacEHS, v. 1, n. 2, Artigo 5. p. 1-10. 2006.

FAKHOURY, N. A. Estudo da qualidade do ar interior em ambientes educacionais. 2017. Dissertação de Mestrado (Mestre em Ciências). Universidade de São Paulo. 198 p.

FERREIRA, D.; REBELO, A.; SANTOS, J. SOUSA, V. SILVA, M. V. Estabelecimentos de restauração e bebidas: estudo sobre a qualidade do ar interior em cozinhas. Occupational Safety and Hygi ene-SHO 2012-Book of Abstracts, p. 189-191, 2012.

INMETRO. Instituto Nacional de Metrologia, Normalização e Qualidade Industrial. Ministério do Desenvolvimento, Indústria e Comércio Exterior. Qualidade do ar em estabelecimentos de uso público e coletivo. Brasília, 2002.

LEMOS, M. M. da S. Avaliação da qualidade microbiológica do ar em cozinhas e zonas de buffet. 2011. Dissertação de Mestrado. Escola Superior de Hotelaria e Turismo do Estoril. 54 p.

MENEGALI, I.; TINÓCO, I. de F. F.; BAÊTA, F. da C.; CECON, P. R.; GUIMARÃES, M. C. de C.; CORDEIRO, M. B. Ambiente térmico e concentração de gases em instalações para frangos de corte no período de aquecimento. Revista Brasileira de Engenharia Agrícola e Ambiental, v. 13, n. 10, p. 984-990, 2009.

SANTANA, N. I. L.; RODRIGUES, G. R. S. Acidentes de trabalho em frigoríficos. Cientefico, v. 29, n. 14, 2014.

SCHIRMER, W. N.; PIAN, L. B.; SZYMANSKI, M. S. E.; GAUER, M. A. A poluição do ar em ambientes internos e a síndrome dos edifícios doentes. Ciência \& Saúde Coletiva, v. 16, p. 3583-3590, 2011.

(cc) $\mathrm{EY}$

Este trabalho está licenciado com uma Licença Creative Commons - Atribuição 4.0 Internacional. 